cells/hpf) in patients with U.urealyticum, though this may be attributed to coexisting NG. Agents isolated from endocervix were Ureaplasma parvum 26(40.6\%), N.gonorrhoeae, 17 (26.6\%), Mycoplasma hominis 11(17.2\%), HSV2, 9(14.1\%), Ureaplasma urealyticum 5(7.8\%), T.vaginalis 4(6.3\%), HSV1 and C.trachomatis, 1 each(1.6\%) and Mycoplasma genitalium $(0 \%)$. Bacterial vaginosis was diagnosed in $14(21.9 \%)$ patients. Multiple, two, three, four and five agents were isolated in 10 , 6, 6 and 1patients respectively. Isolation of M.hominis and U. parvum was significantly associated with bacterial vaginosis ( $p$ value $0.04 \& 0.003$ respectively). Non-usage of condoms predisposed to cervicitis.

Conclusion Few C.trachomatis isolates, absence of M.genitalium and increasing prevalence of HSV, highlights the changing etiological pattern of cervicitis. Though U.parvum and M. hominis are usually commensals their high isolation, association with bacterial vaginosis and propensity to cause diseases in preterm infants and extragenital infections puts forth the need for further studies and regular monitoring of agents implicated in cervicitis.

\section{P370 WHAT ARE THE RISK FACTORS FOR CHLAMYDIA IN WOMEN ATTENDING AN ABORTION SERVICE IN THE UK?}

${ }^{1,2} \mathrm{~F}$ Von Hawrylak*, ${ }^{1} \mathrm{R}$ Johnson, ${ }^{1} \mathrm{~A}$ Liebow, ${ }^{3} \mathrm{P}$ Muir, ${ }^{1} \mathrm{M}$ Singh, ${ }^{1,2} \mathrm{P}$ Horner. ${ }^{1}$ University Hospitals Bristol, Bristol, UK; ${ }^{2}$ Bristol University, Bristol, UK; ${ }^{3}$ Public Health England South West laboratory, Bristol, UK

10.1136/sextrans-2021-sti.409

Background The 2019 updated NICE abortion guidance suggested that prophylactic antibiotics should no longer be routinely given for medical abortions and instead recommended individual risk assessment.

Untreated Chlamydia trachomatis is an important cause of severe infection post abortion. In 2002, 75 (7.5\%) of 998 women having an abortion tested chlamydia-positive using a nucleic acid amplification test (NAAT). Women attending our abortion service have been tested for Chlamydia using a NAAT. We wished to obtain contemporary estimates of Chlamydia prevalence and examine associated risk factors.

Methods Data from 13,427 women aged 15-50 years having an abortion between April 2010 and March 2020, was retrospectively analysed (SPSS, IBM). Ethics approval was sought and all data was anonymised.

Results Of 13,337 with Chlamydia NAAT results available, $4.2 \%$ (565) were Chlamydia-positive. Chlamydia was associated with younger age $(\mathrm{p}<0.0001$, Chi-square $)$ thus $7.1 \%$ $(164 / 2135)$ of $15-19$ year olds, 5.5\% (222/3782) of 20-24 year olds and $2.5 \%(174 / 6774)$ of women over 24 years old were Chlamydia-positive. Chlamydia detection was associated with 2 or more partners within the past year in women aged 20-24 years old, $8 \%(94 / 1181)$ vs $4.6 \%$ (106/2304) $(\mathrm{p}<0.0001$, OR 1.8; 95\%CI, 1.35-2.39) and women over 25 years old, $4.2 \% \quad(53 / 1270)$ vs $2.1 \% \quad(95 / 4507)(\mathrm{p}<0.0001$, OR 2.0 ; 95\% CI 1.44-2.85) but not women aged 15-19 years old, $7.8 \%(50 / 643)$ vs $6.1 \%(83 / 1367)(\mathrm{p}=0.27$ OR 1.3 ; $95 \%$ CI $0.91-1.9)$. For women who had a new partner in the last 3 months, findings were similar regardless of age.

Conclusion Chlamydia prevalence in women undergoing an abortion is lower than that observed in 2002 but is higher than the general population. Although Chlamydiawas associated with 2 or more partners in the previous year in women aged over 19 years, the association is weaker than that observed in the general population and no association was observed with partner change in women aged 15-19 years old

\section{P371 COMBINATION HIV/HCV/HBV/STIS PREVENTION AMONG MSM AND USE OF MOBILE APPLICATIONS/SOCIAL NETWORKS AT THE COVID-19 CONDITIONS IN UKRAINE}

A Chernyshev*, A Bogoslavets, R Marchenko. Alliance.Global, 62B, B.Kchmelnitskogo str., Ukraine

\subsection{6/sextrans-2021-sti.410}

Background/Purpose Restrictions of COVID-19 pandemic have made it more difficult to provide HIV testing and PrEP services to MSM in large Ukrainian cities. The purpose of the intervention is to maintain the effectiveness of the provision of these services during COVID-19 and to implement new/ innovative and more mobile interventions in this regard.

Approach The methodology consists of conducting two national campaigns aimed at MSM recruiting in 3 largest cities of Ukraine, for conducting of HIV/STI/HCV testing, as well as to attract to the PrEP program, through targeted advertising on gay dating applications and social networks for directing to web-resources https://gettest.com.ua and https://prep. com.ua, for passing of testing and/or to receive equal counseling on PrEP, at Alliance.Global's testing points. All confirmed HIV-positive MSM have been provided a social support to receive ART; for HIV-negative MSM, we proposed to become a member of the free PrEP program.

Outcomes/Results Thanks to advertising on two web resources, during September-December 2020, 841 MSM registered for HIV/HCV/STI testing through the GetTest website and $80 \%$ of them were tested (the number of HIV-positive results was approximately 4\%). 3244 MSM have learned about the PrEP program during this period, approximately 300 new MSM have been attracted to the PrEP program (the coverage of the PrEP intervention in 2020 amounted about 1,300 MSM).

Innovations/Conclusions Thanks to the introduction of two advertising campaigns on the Internet, at the COVID-19 conditions, as well as such innovative interventions as delivery of clients by taxi to receive PrEP in a medical institution, receiving the free premium accounts in the mobile gay application Hornet, compliance with sanitary norms (free masks, disinfectants, etc.) and mandatory pre-registration (to avoid queues), we were able to successfully saved services, and increase the intensity of testing and staging to PrEP for MSM compared to the first half of 2020 .

\section{P374 CHARACTERISTICS OF PREGNANT WOMEN, PARTNERS, AND PARTNER TREATMENT PREFERENCES AMONG WOMEN UNDERGOING SEXUALLY TRANSMITTED INFECTION SCREENING, RAWALPINDI, PAKISTAN}

${ }^{1}$ E Hansman* ${ }^{*},{ }^{1} \mathrm{~A}$ Chaudry, ${ }^{2} \mathrm{R}$ Chaudhri, ${ }^{2} \mathrm{~A}$ Kayani, ${ }^{1} \mathrm{~L}$ Hayes, ${ }^{3} \mathrm{C}$ Bristow, ${ }^{2} \mathrm{~K}$ Javaid ${ }^{2} \mathrm{~N}$ Khan, ${ }^{2} \mathrm{~S}$ Akhlaque, ${ }^{2} \mathrm{~B}$ Yasmeen, ${ }^{1} \mathrm{~J}$ Klausner. 'David Geffen School of Medicine, UCLA, Los Angeles, USA; ${ }^{2}$ Rawalpindi Medical University/Holy Family Hospital, Rawalpindi, Pakistan; ${ }^{3}$ University of California San Diego School of Medicine, San Diego, USA 
Background This study sought to characterize dyads of pregnant women and their partners in Rawalpindi, Pakistan, and to understand STI partner notification and treatment preferences in this population.

Methods We enrolled pregnant women seeking antenatal care at Holy Family Hospital from September to December 2019 and performed STI testing. We used interviewer-administered surveys to collect medical, social and sexual histories, and partner treatment preferences. Participants testing positive for STIs and their partners were treated.

Results We enrolled 1001 women seeking antenatal care in Rawalpindi, Pakistan. Of those participants, nearly all were married (99.7\%) and unemployed (95.6\%), while $92.7 \%$ of their partners worked full time. A majority achieved an equal $(38.6 \%)$ or higher $(27.9 \%)$ level of education than their partner. Within these dyads, $40.6 \%$ reported rarely or never being asked for permission before sex, and only 9 participants reported initiating sex $(0.9 \%)$. The majority reported never using condoms before $(87.8 \%)$ or since $(95.5 \%)$ becoming pregnant. Nearly all participants would be comfortable disclosing the results of their STI test to their partner (99.9\%) and would inform their partner of a positive result (98.4\%). Of those who were diagnosed with an STI (11) and were not lost to follow-up (6), 100\% of partners received treatment via expedited partner therapy, although $0 \%$ of partners sought further care.

Conclusion In this population of pregnant women from Rawalpindi, Pakistan, participants were largely married, unemployed, and educated to at least an equal level as their partner. STIs were rare despite lack of consistent condom use, and participants were willing to notify partners of STI results. Nearly half of participants testing positive for an STI were lost to follow-up, raising concerns for adherence; the remainder, however, successfully delivered treatment to their partner, suggesting that antenatal screening programs in this setting would be successful despite low case positivity rates.

\section{P375 PREP CARE CONTINUUM: PERCEPTIONS AND EXPERIENCES OF BLACK SAME-GENDER-LOVING MEN FROM A SOUTHERN U.S. CITY}

A Corneli* ${ }^{*}$ B Perry, T Munn, M Hunter. Duke University, Durham, USA

\subsection{6/sextrans-2021-sti.412}

Introduction U.S. HIV/AIDS Strategy has prioritized men who have sex with men (MSM) and people in the Southern U.S. as key populations for HIV prevention interventions. We-a community-academic partnership - conducted formative research on the PrEP care continuum with Black same-genderloving men (BSGLM) in a Southern U.S. city.

Methods We conducted six focus group discussions (FGDs) with 35 BSGLM; most had never used PrEP. We explored PrEP awareness and acceptability - the continuum's first stage. We also conducted 18 in-depth interviews (IDIs) with BSGLM who have taken or discussed PrEP with a provider. The IDIs focused on PrEP uptake-the continuum's second stage-and adherence and retention in care-the final stage. All FGDs/ IDIs were recorded, transcribed, and analyzed using applied thematic analysis.

Results FGD - participants said BSGLM are generally aware of PrEP, but misinformation exists and knowledge of PrEP-related services is limited. Perceived risk influences PrEP interest-participants explained that BSGLM consider their receptive or insertive role when evaluating HIV vulnerability and BSGLM who do not identify as gay are less interested in PrEP. Fears are also common, including fear of facing consequences of past risky behaviors, others learning of one's PrEP use, and talking to providers about sexual behaviors. Participants also described existing discrimination and stigma toward BSGLM from multiple sources, including the Black community and the broader MSM community, and concerns about PrEPrelated stigma. IDI-participants said PrEP provides a 'peace of mind' but acknowledged challenges with pill taking. Several also described experiencing PrEP-related stigma as well as stigma from the Black community for being a Black gay man. Conclusion We need multi-level interventions that support BSGLM at each stage of the PrEP care continuum. BSGLM need information to make informed decisions about PrEP and supportive community environments to use PrEP for as long as risk persists.

\section{P377 CHLAMYDIA TRACHOMATIS AND NEISSERIA GONORRHOEAE DNA STABILITY IN PRESERVED FIRST-VOID URINE}

${ }^{1} \mathrm{M}$ Laeremans, ${ }^{2} \mathrm{~L}$ Mahieu, ${ }^{1} \mathrm{D}$ Pasmans, ${ }^{1} \mathrm{~K}$ Beyers, ${ }^{3} \mathrm{E}$ Padalko, ${ }^{2} \mathrm{P}$ Cos, ${ }^{1} \mathrm{~V}$ Vankerckhoven*. 'Novosanis, Wijnegem, Belgium; ' ${ }^{2}$ University of Antwerp, Antwerp, Belgium; ${ }^{3}$ University Hospital Ghent, Ghent, Belgium

\subsection{6/sextrans-2021-sti.413}

The aim of this study was to evaluate the performance of UCM (Urine Conservation Medium, Novosanis) for Chlamydia trachomatis (CT) and Neisseria gonorrhoeae (NG) DNA preservation at room temperature (RT).

To compare the stability of CT and NG DNA in unpreserved and UCM-preserved samples, five urine samples were spiked to obtain $99 \mathrm{CT}$ and $75.5 \mathrm{NG}$ DNA copies/ $\mu \mathrm{L}$ in the final aliquot. For UCM-preserved samples, UCM was spiked and urine was added in a 2:1 (urine:UCM) ratio to mimic real-life use of Colli-Pee containing UCM. A total of 30 aliquots were analyzed using the Abbott Real-Time CT/NG assay after storage at RT for 1,8 and 15 days. Mixed-effect regression analysis was used to estimate the effect of UCM on CT and NG Ct values at each timepoint, with urine samples as random effects.

After 15 days of storage, all UCM-preserved samples were positive for CT and NG, while only one and three unpreserved samples remained positive for CT and NG respectively. $\mathrm{Ct}$ values where samples were negative for $\mathrm{CT}$ and/or $\mathrm{NG}$ were defined as the cut-off values (cut-off $8 \mathrm{~d}$ : $\mathrm{CT}=39.66$, $\mathrm{NG}=39.93$; cut-off $15 \mathrm{~d}$ : $\mathrm{CT}=39.56, \mathrm{NG}=39.92$ ). At each timepoint, Ct-values for CT and NG were significantly lower for preserved compared to unpreserved samples. At 1, 8 and 15 days of storage UCM-preserved samples showed an estimated differences of $-6.03,-5.91$ and -4.60 cycles for CT, and $-6.91,-7.20$ and -7.36 for NG respectively. All effects are statistically significant with $\mathrm{p}<0.01$.

UCM-preserved urine sampling enables preservation of CT and NG DNA for 15 days of storage at RT. The use of Colli-Pee, whereby the collector tube is prefilled with UCM, allows for immediate mixing of urine and preservative. This offers opportunities for home-based testing where sample quality is maintained during storage and sample shipment to the laboratory by regular postal mail. 